\title{
Biosimilars naming and prescribing policy in Australia
}

\author{
Brendan Shaw, PhD
}

To the Editor:

I wanted to bring to your attention the following paper which was published in Volume 2/Year 2013/Issue 3 of the Generics and Biosimilars Initiative Journal.

The Perspective paper titled Licensing and prescribing biosimilars in Australia [1], Professor David Power's description of the Australian reimbursement system contains a number of factual errors. Left uncorrected those errors lead people to misunderstand the market for biosimilars in Australia.

Professor Power erroneously draws a link between Australia's policies on substitution at a pharmacy level and Australia's naming policies for medicines. He states:

"Where the products have the same INN names, as for filgrastim, and the prescriber uses the generic drug name, the pharmacist is free to choose any of the products with that name.'

\begin{abstract}
And later:
'Since PBS [Pharmaceutical Benefits Scheme] does not permit automatic substitution of biosimilars with different INN, pharmacists are unable to substitute a glycosylated biosimilar for its (presumably more expensive) comparator drug. Where the drug has the same INN, then the cheapest product can be supplied unless the prescribers stipulate use of a particular brand.'
\end{abstract}

This is incorrect. While all available filgrastim biosimilars in Australia have identical non-proprietary names as the reference or originator product, i.e filgrastim, none are considered 'substitutable' within the context of Australia's National Health Act.

As Professor Power correctly notes, the Pharmaceutical Benefits Advisory Committee (PBAC) determines which molecules may or may not be substituted at a pharmacy level. Those brands of medicines deemed substitutable at a pharmacy level by the PBAC include a notation in the official Pharmaceutical Benefits Schedule known as an 'a-flag'. The PBAC generally accepts the Australian regulator's (Therapeutic Goods Administration) assessment that a molecule is bioequivalent to its reference product as the principal criterion for 'a-flagging'. The PBAC also considers other factors, including whether medicines have a narrow therapeutic index.

In the case of the filgrastim biosimilar, Nivestim, the PBAC rejected a request from the sponsor for an 'a-flag'. The PBAC's publicly available recommendation on the listing of Nivestim states:

'SBMPS (similar biological medicinal products, aka biosimilars) havesomeconceptual parallels with generic versions of products containing chemically-derived small molecules as the active substances. However, although small molecule generic products may be approved for marketing on the basis of bioequivalence (or in limited cases, therapeutic equivalence) to a reference product, these concepts may not at this time be extrapolated to SBMPS. Proteins and other biological medicinal products can be more complex than chemically synthesised medicines. This is in part because even highly purified protein products may consist of more than one molecular entity, and are usually mixtures of many closely related molecular species. This within product microbeterogeneity may be substantial. Thus, even though a SBMP will have the same encoding DNA sequence as the reference product, the two products may differ in other key attributes.

\section{PBS Listing of SBMPs}

The current practice of ' $a$ ' flagging in the Schedule of Pharmaceutical Benefits, denoting that brand substitution may be undertaken by pharmacists at the point of dispensing, will not be applied to SBMPS at this time unless a Therapeutic Goods
Administration (TGA) issued statement supportive of 'a' flagging is available' [2].

The PBAC concluded:

'The PBAC recommended listing of the requested filgrastim products, noting that the TGA delegate proposed to register the products as similar biological medicinal products being of 'comparable efficacy and safety' and having the same nonproprietary name as its reference product on the basis of an abridged dataset according to the European Union guidelines adopted by the TGA....

The PBAC recommended that ' $a$ ' flagging for the purposes of subsection 103(2 A)(b) of the National Health Act 1953 should not be applied across the two sets of filgrastim products, noting the absence of a TGA issued statement, at the time of consideration, that would support 'a' flagging'.

Thus, contrary to Professor Power's statement pharmacists in Australia cannot 'freely substitute' biological medicines with the same INN.

Significantly, the recently released TGA guidelines on [evaluation of] biosimilars (www. tga.gov.au/industry/pm-argpm-biosimilars-00. htm) reaffirm the Australian regulator's view about the inappropriateness of pharmacy level substitution for these products. In these guidelines, TGA has indicated that the product information will include words similar to the following:

'The level of comparability that has been shown is not sufficient to designate this product as a generic version of [Reference product name]. Replacement of [Reference product name] with [Biosimilar product name], or vice versa, should take place only under the supervision of the prescribing medical practitioner.'

TGA has also advised that the accompanying approval letter will include words similar to the following:

'It is the TGA's view that it is not currently possible to determine a degree of similarity, between a biosimilar and an already registered biological medicine sufficient to support a designation by the TGA of 'bioequivalence'.'

On this basis, it is unlikely that the PBAC will permit an 'a-flagging' of a biosimilar

Author: Brendan Shaw, PhD, Chief Executive, Medicines Australia Ltd, Level 1, Napier Close, Deakin Act 2600, Australia

Submitted: 31 October 2013; Revised: 6 November 2013; Accepted: 11 November 2013; Published online first: 25 November 2013 
soon, regardless of whether the biosimilar was registered in Australia with identical INN or a unique non-proprietary name.

The TGA's new naming convention, requiring distinguishable names for biosimilar medicines, and its policy on substitution are separate and distinct issues, and should be treated as such.

Professor David Power's reply:

I wish to thank Dr Shaw for his reply and for correcting any misleading statements in my article. It was written very much from the viewpoint of an end-user of the system. In that vein, it is still not clear to me how a pharmacist decides what product to provide when the practitioner prescribes by INN name if there are two or more products available with that name. Perhaps that could also be clarified by Dr Shaw.

Dr Brendan Shaw's reply:

This is one of the motivations for the Australian regulators move to create a distinguishable naming policy for biosimilars.
The pharmacist currently has to rely on brand name prescribing or otherwise contact the prescriber if there are no continuing records. The Australian regulator has issued information for bealth professionals (copy provided as below) [3] stating clearly that ...

'Biosimilars should be prescribed by name: Unlike generic small-molecule medicines, which can simply be specified using the international nonproprietary name (INN), biosimilars - and indeed all biological medicines - should be clearly distinguishable on any prescription (preferably by using both trade name and non-proprietary name).

Unlike generic medicines, which must demonstrate bioequivalence to the reference product, biosimilars are not bioequivalent to the products they follow and as such pharmacists may not substitute a biosimilar for the original medicine. This is why it is important to specify which product you are proposing for your patient.'
Competing interests: Dr Brendan Shaw is the CEO of Medicines Australia, the peak body representing the innovative pharmaceutical industry in Australia. Dr Shaw declares no other competing interests.

Provenance and peer review: Not commissioned; internally peer reviewed.

\footnotetext{
References

1. Power D, Licensing and prescribing biosimilars in Australia. Generics and Biosimilars Initiative Journal (GaBI Journal). 2013:2(3):152-4. doi:10.5639/gabij. 2013.0203.030

2. Australian Government. The Department of Health. Therapeutic Goods Administration. Public Summary Document. November 2010 PBAC Meeting. Available from: http://www.health.gov.au/internet/main/ publishing.nsf/Content/pbac-psd-filgrastim-nov10

3. Australian Government. The Department of Health. Therapeutic Goods Administration. Health professional information and education. Biosimilar medicines - information for healthcare professionals. 5 November 2013. Available from: http://www.tga gov.au/hp/hp-information-biosimilars.htm
} DOI: 10.5639/gabij.2013.0204.048

Copyright (C) 2013 Pro Pharma Communications International 